\title{
LESSONS LEARNT IN THE COVID-19 PANDEMIC- MULTISYSTEM INFLAMMATORY SYNDROME IN CHILDREN (MIS-C)
}

\author{
Nagendra Chaudhary ${ }^{1}$, Shatdal Chaudhary ${ }^{2}$ \\ ${ }^{1}$ Department of Pediatrics, Universal College of Medical Sciences, Bhairahawa, Nepal \\ ${ }^{2}$ Department of Internal Medicine, Universal College of Medical Sciences, Bhairahawa, Nepal
}

The recent ongoing pandemic of corona virus disease (COVID-19) is caused by the novel corona virus which was first identified in 2019 in China. The virus causing COVID-19 disease is called severe acute respiratory syndrome coronavirus 2 (SARS-CoV-2). ${ }^{1}$ Although the signs and symptoms of COVID-19 are mild in children, in rare cases, they can have severe clinical presentations. The actual incidence of multisystem inflammatory syndrome in children (MIS-C) is not known but available data suggests it to be relatively rare $(<1 \%){ }^{2}$. The first report of MIS-C was from the UK in a series of eight children at a tertiary centre in South East England. ${ }^{3}$ Cases were then after reported from other parts of the world (Europe, Canada, the United States, and South Africa). Also, there have been few reports of MIS-C from China and other Asian countries with high rates of COVID-19 early in the pandemic. ${ }^{4}$

World health organisation (WHO) and Centers for disease control and prevention (CDC), both have published the case definitions for MIS-C with few differences ${ }^{4}$. Both definitions require fever (duration of fever varies in both definitions), elevated inflammatory markers, at least two signs of multisystem involvement, evidence of SARS-CoV-2 infection or exposure, and exclusion of other potential causes. The CDC case definition requires that the child have severe symptoms requiring hospitalization, whereas the WHO case definition does not. These definitions are likely to change as more information becomes available in future. Recently, in April 2021, the American College of Rheumatology (ACR) has also revised its clinical guidelines for diagnosing and managing hyper inflammation and MIS-C.

The exact pathophysiology of MIS-C is still not clear but is hypothesized that immune dysregulation plays an important role. The syndrome is supposed to result from an abnormal immune response to the virus, with some clinical similarities to Kawasaki disease (KD), macrophage activation syndrome (MAS), and cytokine release syndrome..$^{2-3}$ However, based on the available studies, MIS-C appears to have an immunophenotype that is distinct from $\mathrm{KD}$ and MAS. Preliminary studies suggest that patients with severe MIS-C have persistent immunoglobulin $\mathrm{G}$ ( $\mathrm{IgG}$ ) antibodies with enhanced ability to activate monocytes, persistent cytopenias (particularly $\mathrm{T}$ cell lymphopenia), and greater activation of CD8+ $\mathrm{T}$ cells that differ from findings in acute COVID-19 infection. Many affected children have negative polymerase chain reaction (PCR) testing for SARS-CoV-2 but have positive serology, a finding that further supports the hypothesis that MIS-C is related to immune dysregulation occurring after acute infection has passed. ${ }^{6}$

In children who have a known history of documented or suspected COVID-19, the usual duration between acute infection and onset of MIS-C symptoms is two to six weeks. However, rare cases of MIS-C occurring $>6$ weeks after the acute SARS-CoV-2 infection have been reported. In many cases, the duration of time between acute infection and onset of MIS-C symptoms is unknown because the child was asymptomatic at the time of acute infection. Although clinical diagnostic and management guidelines are being developed, the diagnosis of MIS-C remains challenging because children can present with non-specific symptoms. ${ }^{6}$

Common manifestations of MIS-C in children are fever, gastrointestinal symptoms (vomiting, diarrhea, and abdominal pain), rash, conjunctivitis, mucous membrane involvement (red or swollen lips, strawberry tongue), neurocognitive symptoms (headache, lethargy, and confusion), respiratory symptoms, sore throat, myalgia, swollen legs/hands, and lymphadenopathy. Complications like myocardial dysfunction, arrhythmia, respiratory failure, acute kidney injury, serositis, hepatitis and meningo encephalitis can occur in these children. Children having any of these complication are graded as moderate to severe MIS-C.

All patients with suspected MIS-C should be tested for SARSCoV-2, including both serology and reverse transcription PCR (RT-PCR) on a nasopharyngeal swab. The challenges on the diagnosis of MIS-C are due to rapidly changing strains of SARS-Co-2. ${ }^{7}$

Approximately $60 \%$ of patients have positive serology with negative PCR, and approximately $30-35 \%$ are positive on both tests. Around $5-10 \%$ cases have negative results on both tests where the diagnosis of MIS-C requires an epidemiologic link to SARS-CoV-2 (e.g., exposure to an individual with known COVID-19 within the four weeks prior to the onset of symptoms). Laboratory parameters supporting the diagnosis of MIS-C are abnormal blood counts (mild anemia, lymphocytopenia, neutrophilia and thrombocytopenia), raised inflammatory markers (CRP, ESR, d-dimer, fibrinogen, ferritin, procalcitonin and IL-6), elevated cardiac markers

DOI: https://doi.org/10.3126/jucms.v9i02.41939 
(troponin, BNP and pro-BNP), hypoalbuminemia, mild elevation of liver enzymes, raised LDH, and hypertriglyceridemia. Cardiac involvement is common in MIS-C and echocardiography generally shows depressed LV function, coronary artery dilatation or aneurysm, mitral regurgitation or pericardial effusion.

Almost all MIS-C cases require admissions to pediatric intensive care unit. Treatment is focussed to address the complications present in the child. Most children with moderate to severe manifestations are treated initially with intravenous immune globulin (2 gm/ $\mathrm{kg}$ over $8-12$ hours), glucocorticoids and biologics but the results of clinical trials are still awaited. $^{?}$

If IVIG is not available, treatment with glucocorticoids alone is a reasonable option. Patients presenting with severe multisystem involvement and shock should receive empirical broad-spectrum antibiotic therapy till the culture results are awaited $^{5}$. The role of SARS-CoV-2 antiviral therapies (e.g., remdesivir) in the management of MIS-C has not been well studied, but such therapies are rarely required, unless there are overlapping clinical features due to COVID-19. One of the main ongoing trials "the UK RECOVERY trial (NCT04381936)", supported by the Bill and Melinda Gates Foundation is due for completion in December 2021 and is one of the few trials randomly assigning patients with MIS-C to the biologics, tocilizumab or anakinra.

Therefore, in the current situation, there are several challenges ahead for preventing, diagnosing, and managing MIS-C especially in low resource countries like Nepal as the diagnosis and management protocols are continuously being updated. Any children suspected of MIS-C should be timely referred to a specialist without any further delay. Results of many ongoing trials will further enrich our knowledge and help us to diagnose and treat MIS-C more effectively.

\section{REFERENCES}

1. Riphagen S, Gomez X, Gonzalez-Martinez C, et al. Hyper inflammatory shock in children during COVID-19 pandemic. Lancet. 2020; 395:1607-8

2. Godfred-Cato S, Bryant B, Leung J, et al. California MIS-C Response Team. COVID-19-associated multisystem inflammatory syndrome in children - United States, March-July 2020. Morb Mortal Wkly Rep. 2020;69(32):1074-80

3. Verdoni L, Mazza A, Gervasoni A, et al. An outbreak of severe Kawasaki-like disease at the Italian epicentre of the SARSCoV-2 epidemic: An observational cohort study. Lancet. 2020; 395:1771-78

4. Dufort EM, Koumans EH, Chow EJ, et al. New York State and CDC Multisystem Inflammatory Syndrome in Children
Investigation Team. Multisystem inflammatory syndrome in children in New York State. N Engl J Med. 2020;383:347-58

5. Henderson LA, Canna SW, Friedman KG, et al. American College of Rheumatology clinical guidance for multisystem inflammatory syndrome in children associated with SARSCoV-2 and hyperinflammation in pediatric COVID-19: Version 2. Arthritis Rheumatol. 2021;73(4)

6. Centers for Disease Control and Prevention (CDC). Multisystem inflammatory disease in children (MIS-C). May 2021.

7. Davies P. Addressing fundamental questions on MIS-C. Lancet Child Adolesc Health. 2021;5(5):310-11 